\title{
Delayed bipedicled nasolabial flap in facial reconstruction
}

\author{
Cindy Siaw-Lin Goh ${ }^{1}$, Joshua Guy Perrett ${ }^{2}$, Manzhi Wong ${ }^{1}$, Bien-Keem Tan ${ }^{1}$ \\ ${ }^{1}$ Department of Plastic, Reconstructive and Aesthetic Surgery, Singapore General Hospital, Singapore, Singapore; ${ }^{2}$ Department of General \\ Surgery, Waitemata District Health Board, Auckland, New Zealand
}

Background The nasolabial flap is ideal for reconstruction of the nasal alar subunit due to its proximity, color and contour match, and well-placed donor scar. When raised as a randompattern flap, there is a risk of vascular compromise to the tip with increased flap length and aggressive flap thinning. Surgical delay can greatly improve the chances of tip survival, allowing the harvest of longer flaps with greater reach.

Methods We describe our technique of lengthening the nasolabial flap through multiple delay procedures. A bipedicled flap was first raised and then transferred as a unipedicled flap with a 6:1 length-to-width ratio. During the delay process, the flap tip was thinned to the subdermal layer.

Results In our case series of seven patients, defects as far as the medial canthal area and contralateral ala were reconstructed successfully with no incidence of tip necrosis or flap loss. The resultant flaps were thin enough to be folded over for the reconstruction of alar rim defects.

Conclusions We highlight the success of our surgical technique in creating thin and robust nasolabial flaps for the reconstruction of full-thickness defects around the nose.

Keywords Reconstructive surgical procedures / Surgical flaps / Nasolabial fold / Graft survival
Correspondence: Bien-Keem Tan Department of Plastic, Reconstructive and Aesthetic Surgery, Singapore General Hospital, Outram Road, Singapore 169608, Singapore

Tel: $+65-6321-4686$

Fax: +65-6227-3573

E-mail: bienkeem@gmail.com

We thank Evan Lim Hock Tat for his assistance with the design of the medical illustration (Fig. 4, original creation).

\section{INTRODUCTION}

The nasolabial flap is ideal for the reconstruction of defects involving the nose and cheek. It provides good soft tissue color and texture match due to its proximity to those areas and results in a well-camouflaged scar in the nasolabial crease. However, due to vascularity concerns, its reach is traditionally limited to the lower-third nasal subunits, dorsum, and adjacent cheek area. Aggressive thinning of the flap is often required for a thin and pliable reconstruction, but poses a risk of necrosis of the tip, which is the part with the greatest utility.
Surgical delay can greatly improve flap survival, allowing the safe harvest of longer flaps with greater reach [1]. The use of a bipedicled design, one method of surgical delay, permits the elevation of random-pattern flaps with greater length-to-width ratios [2]. Here, we describe our technique of lengthening the nasolabial flap through multiple delay procedures. The flap was initially raised as a bipedicled flap and then transferred as a superiorly-based unipedicled flap to reach distant defects around the nose. The delay process allowed the tip to be thinned sufficiently to create "fold-over" flaps for the reconstruction of fullthickness alar rim defects. 


\section{METHODS}

A retrospective case note review was conducted of all patients who underwent facial reconstruction using a delayed bipedicled flap. The study was approved by the SingHealth Institutional Review Board (IRB No. 2017/2444). All patients provided informed consent for the surgical procedures and further consent was obtained from the three cases presented below for publication of their images. Patient demographics, clinical characteristics, and postoperative outcomes were documented.

\section{Surgical technique}

A narrow flap with superior and inferior pedicles was designed along the nasolabial crease, extending down to the jawline. The width of the flap was limited by the ability to close the donor site primarily, and the flap was gently tapered towards its distal end. During the first stage of surgery, the flap was raised in the subcutaneous plane in a bipedicled fashion. The donor site was closed beneath the flap to prevent revascularization of its undersurface. In the first three cases, the undersurface of the flap was dressed regularly with tulle gras to keep it clean and moist. In the later four cases, the entire exposed raw undersurface of the flap was grafted with a split-thickness skin graft from the thigh. This acted as a biological dressing to prevent flap infection, which could have led to scarring or flap loss. The grafted aspect could also later be used to reconstruct the internal nasal lining.

Over 2 weeks, the flap tip was "trained" in the outpatient setting by progressively reducing the width and thickness of the distal pedicle every few days until it was thinned to the subdermal layer. Staged division in this fashion avoided tip ischaemia and congestion. Once distal division was complete, the tip was partially inset into the defect and the proximal incisions were extended as necessary to lengthen the flap to avoid tension. The proximal pedicle was then progressively divided across its width over the next 2 weeks at a point distal to its origin to maximize the survival of the bridging portion. This provided sufficient length for the flap to be folded over on itself during alar rim reconstruction. With the completion of flap division, flap inset was finalised and the proximal remnant flap tissue was returned to the nasolabial area to prevent nasal deviation. In select patients, conchal or rib cartilage grafts were harvested to provide support for the reconstruction. The entire process took approximately a month to complete.

\section{RESULTS}

Between January 2004 and December 2014, seven patients (five females, two males) underwent facial reconstruction using the above-described technique (Table 1). Their mean age was 60.1 years (range, 31-84 years). The defects were caused by tumour extirpation in five patients and trauma in two patients. Five patients had nasal ala defects, whilst two patients had defects isolated from and distant to the ala. The median follow-up duration was 32 months (range, 12-120 months). Flap length-towidth ratios ranging from 5:1 to 6:1 were achieved in all cases. The average flap length was $6.2 \mathrm{~cm}$ (range, $5.5-6.7 \mathrm{~cm}$ ). Two patients had combined nasolabial and paramedian forehead flaps for the reconstruction of large full-thickness hemi-nasal defects. In these cases, the nasolabial flap was used to recreate the internal lining, whilst external nasal resurfacing was achieved using the forehead flap. Cartilage grafts were used in three cases.

All transferred flaps survived without necrosis or infection. Minor alar slump developed in two patients in whom no support grafts were used, but they both declined further surgery. Four patients underwent minor revision procedures (scar revision or further flap debulking). All patients were subjectively

Table 1. Patient demographics and reconstructive details

\begin{tabular}{|c|c|c|c|c|c|c|c|c|}
\hline $\begin{array}{l}\text { Patient } \\
\text { no. }\end{array}$ & Age (yr)/sex & Defect region & Aetiology & $\begin{array}{l}\text { Defect size } \\
\quad(\mathrm{cm})\end{array}$ & Reconstruction & $\begin{array}{l}\text { Length-to- } \\
\text { width ratio }\end{array}$ & $\begin{array}{c}\text { Cartilage } \\
\text { graft }\end{array}$ & $\begin{array}{c}\text { Revision } \\
\text { procedures }\end{array}$ \\
\hline 1 & $70 / F$ & Ala, cheek & $\mathrm{BCC}$ & $2 \times 1$ & $\begin{array}{l}\text { Contralateral nasolabial flap, } \\
\text { skin graft }\end{array}$ & $6: 1$ & - & Scar revision \\
\hline 2 & $72 / \mathrm{M}$ & Ala & SCC & $2 \times 1.5$ & Ipsilateral nasolabial flap & $5: 1$ & - & Scar revision \\
\hline 3 & $44 / F$ & Medial canthus & $\begin{array}{l}\text { Olfactory } \\
\text { neuroblastoma }\end{array}$ & $2 \times 1$ & Ipsilateral nasolabial flap & $6: 1$ & - & - \\
\hline 4 & $77 / F$ & Ala & BCC & $2 \times 1.5$ & Ipsilateral nasolabial flap & $5: 1$ & Conchal & - \\
\hline 5 & $31 / F$ & Hemi-nasal & Trauma (scar) & $2.5 \times 2$ & $\begin{array}{l}\text { Ipsilateral nasolabial \& } \\
\text { paramedian forehead flaps }\end{array}$ & $5: 1$ & Conchal, rib & $\begin{array}{l}\text { Scar revision, forehead } \\
\text { flap debulking }\end{array}$ \\
\hline 6 & $42 / \mathrm{M}$ & Hemi-nasal & Trauma (loss) & $3 \times 2.5$ & $\begin{array}{l}\text { Ipsilateral nasolabial \& } \\
\text { paramedian forehead flaps }\end{array}$ & $5: 1$ & Conchal & Scar revision \\
\hline 7 & $84 / F$ & Cheek adjacent to al & $B C C$ & $2.5 \times 2$ & Contralateral nasolabial flap & $6: 1$ & - & - \\
\hline
\end{tabular}


satisfied with the final results.

We present three cases to illustrate the utility of this technique.

\section{Case 1}

A 70-year-old woman (patient 1) presented with a right nasolabial fold invasive nodular basal cell carcinoma. Following wide excision, there was a defect of the cheek measuring $4 \times 3 \mathrm{~cm}$ and a $2 \times 1 \mathrm{~cm}$ full-thickness loss of the alar base and nostril floor (Fig. 1).

The cheek wound was resurfaced by local advancement and skin grafting. To reconstruct the alar base, a delayed bipedicled contralateral nasolabial flap with a 6:1 length-to-width ratio was used. The tip was inset into the nostril using flap skin to resurface the internal lining of the ala and nasal floor. The flap was later folded over on itself to complete the reconstruction of the external alar rim.
Six weeks later, the patient underwent bilateral nasolabial fold scar revision. Despite minor asymmetry at 5 years, she was satisfied with the outcome and declined further revision procedures.

\section{Case 2}

A 44-year-old woman (patient 3) was diagnosed with a left-sided olfactory neuroblastoma involving the orbit, nasal cavity, maxilla, and anterior cranial fossa. After neoadjuvant chemo-radiotherapy, she underwent craniofacial resection via a WeberFerguson incision and the bicoronal approach. This was complicated by postoperative wound dehiscence, which left a fullthickness medial canthal defect measuring $2 \times 1 \mathrm{~cm}$ (Fig. 2). Considering the previous degloving of her forehead tissue, possible disruption of the supratrochlear pedicle, and the presence of underlying metallic hardware, the option of a forehead flap was deemed unreliable.

Fig. 1. Case 1

A 70-year-old woman (patient 1) with a right cheek, alar base, and nostril floor defect following wide excision of a right nasolabial basal cell carcinoma. (A) A bipedicled nasolabial flap with a 6:1 length-to-width ratio was raised for nasal reconstruction, whilst the cheek area was advanced and skin-grafted. (B) Inset of the flap was staged by first inserting the tip into the nostril. (C) The proximal pedicle was divided in stages across its width at a point distal to its origin (D) At the final inset, the thinned distal portion of the flap was folded over onto itself to reconstruct the alar rim, whilst the proximal remnant flap tissue was returned to the donor site to prevent nasal deviation. (E-G) Photographs taken 5 years postoperatively. Mild alar slump is evident on a worm's eye view. She had no associated obstructive symptoms and declined further revision with cartilage support grafts.
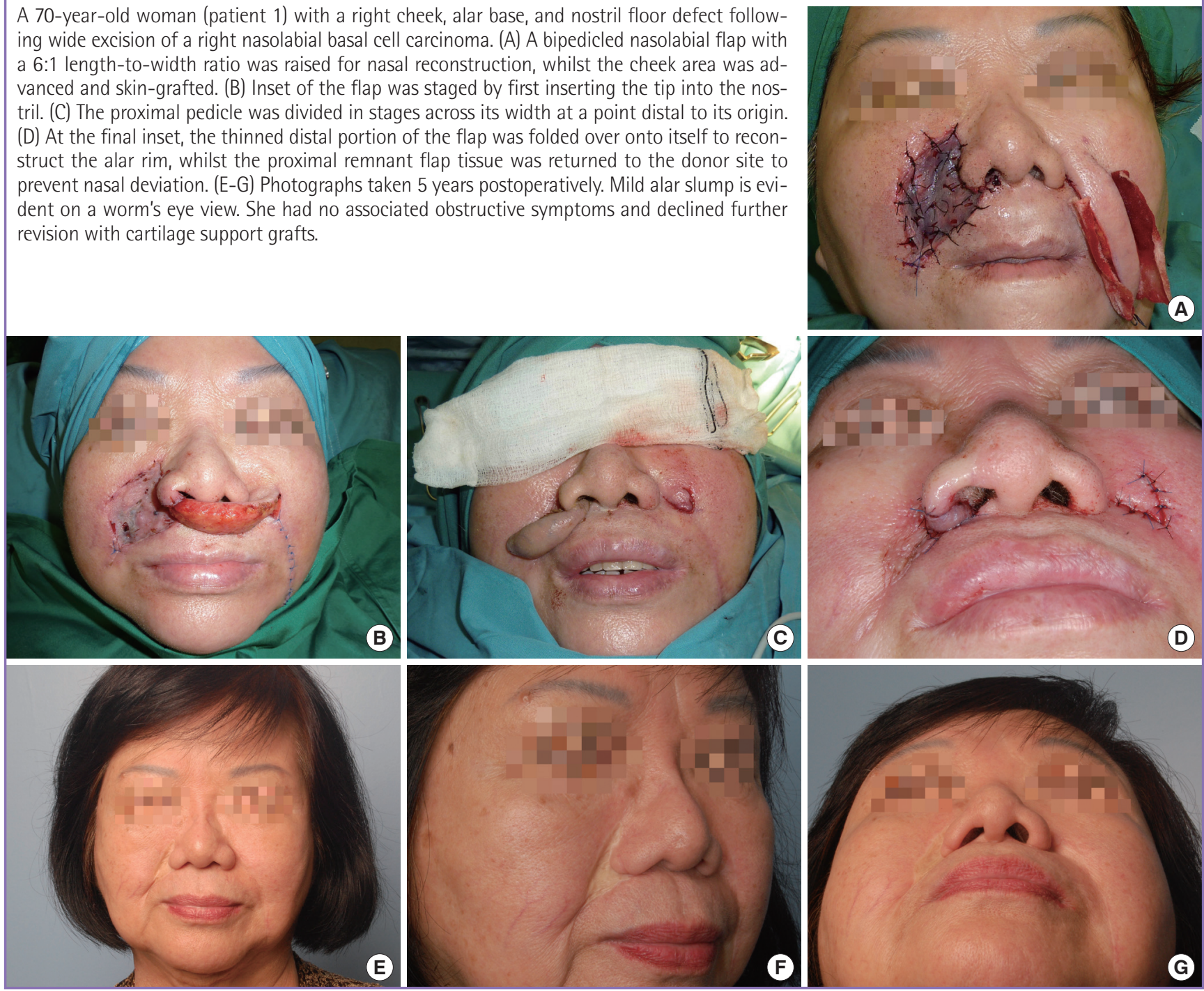


\section{Fig. 2. Case 2}

A 44-year-old woman (patient 3) with dehiscence of a Weber-Ferguson incision that resulted in a left medial canthal defect. (A) An "ultra-long" nasolabial flap was raised in a bipedicled fashion and delayed. The raw undersurface of the flap was skin-grafted. (B) The flap was rotated upwards and the pre-grafted tip surface was used to reconstruct the vestibular lining. (C) Photograph taken 10 years after reconstruction.
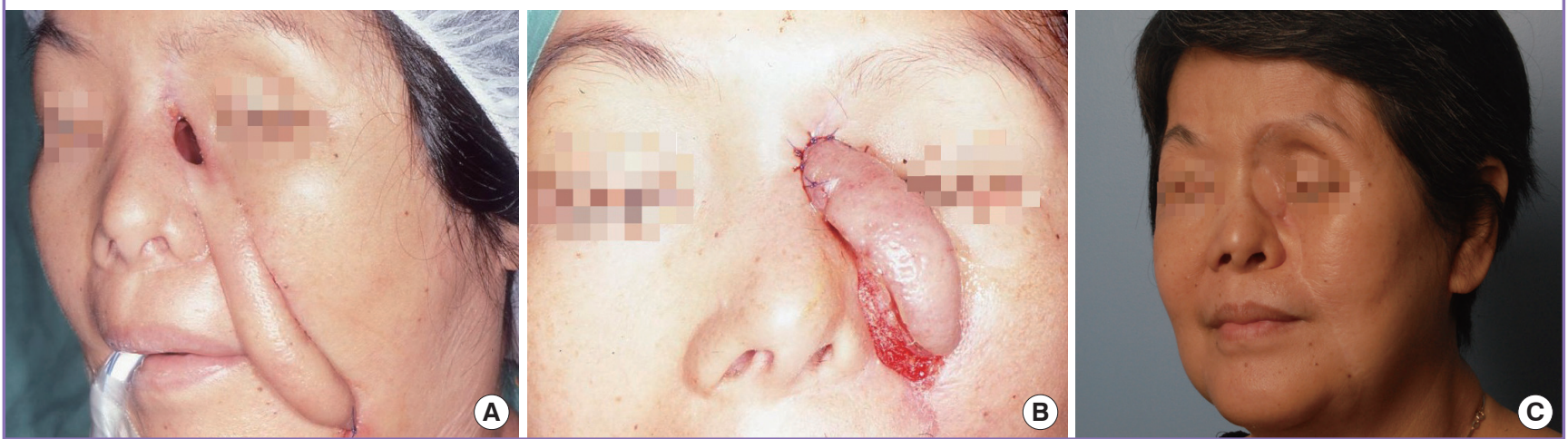

\section{Fig. 3. Case 3}

(A) A 42-year-old male (patient 6) with a traumatic hemi-nasal defect. (B) A bipedicled nasolabial flap was raised and the inferior pedicle was progressively divided to provide a thin and pliable tip. The flap tip was inserted into the nostril using the skin of the flap to reconstruct the internal nasal lining. The external skin defect was resurfaced with an oblique forehead flap. Alar rim support was provided by a conchal cartilage batten graft. $(C, D)$ Photographs taken 4 years after reconstruction.
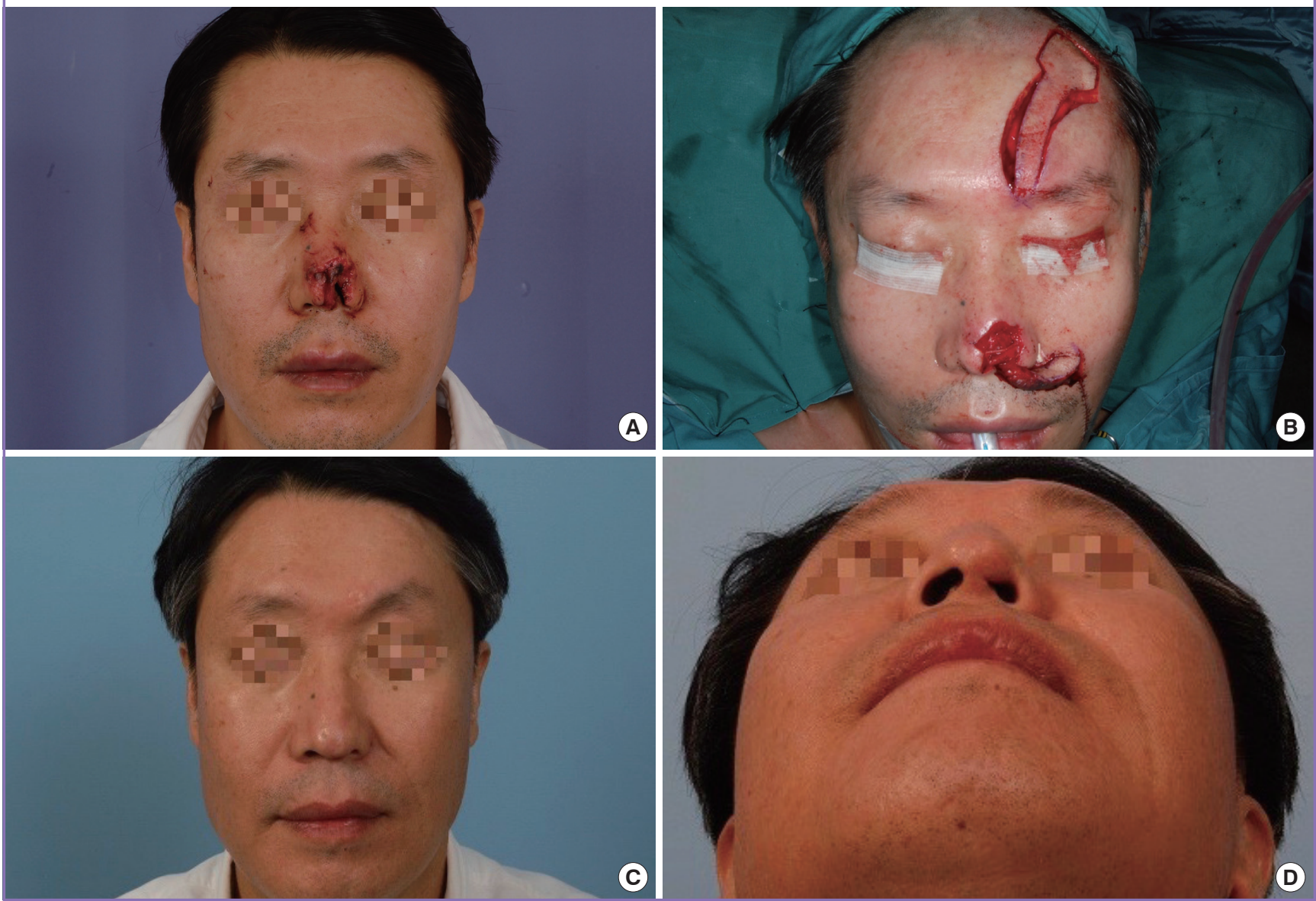

A delayed bipedicled ipsilateral nasolabial flap with a $6: 1$ length-to-width ratio was raised and its undersurface was skingrafted. Following staged division of the inferior pedicle, the flap was transposed medially and upwards into the defect using the pre-grafted undersurface to reconstruct the nasal mucosa. The final inset was completed 2 weeks later. 
The outcomes remained stable at 10 years of follow-up, and the patient was satisfied with the results.

\section{Case 3}

A 42-year-old man (patient 6) presented with a traumatic fullthickness hemi-nasal defect measuring $3 \times 2.5 \mathrm{~cm}$ (Fig. 3). The wound was debrided and temporarily covered with a split-thickness skin graft. Definitive reconstruction was delayed until wound healing was complete.

A bipedicled ipsilateral nasolabial flap with a 5:1 ratio lengthto-width ratio was raised and skin was grafted along its raw undersurface. The tip of the flap was delayed and turned into the nostril to allow replacement of the mucosal lining with flap skin. An oblique forehead flap was raised and transposed inferiorly to resurface the external skin defect. A $2 \times 0.7 \mathrm{~cm}$ conchal cartilage graft was used as a batten graft to support the alar rim. The forehead donor site was closed primarily except at its superior portion, which was left to heal by secondary intention.

Both flaps were progressively delayed, with completion of flap division and final inset 1 month after the initial stage of reconstruction. Scar revision was performed using Z-plasties. The patient was satisfied with the final result and did not report symptoms of nasal obstruction.

\section{DISCUSSION}

The nasolabial flap is a versatile flap that is most often used for reconstruction of the lower-third nasal subunits and dorsum $[3,4]$. It matches the skin characteristics of the nose and cheek well, and provides sufficient soft tissue for the coverage of moderately-sized defects. The resultant donor scar is well-hidden in the nasolabial crease.

The nasolabial flap is supplied by perforating branches of the facial and angular arteries that freely anastomose to supply a rich subdermal plexus. Although the main artery is not normally included in the flap, the small vessels of this plexus are generally oriented along the long axis of the flap, giving it a "degree of axiality" [5]. Length-to-width ratios of 3:1 or less have traditionally been advocated to avoid compromising the vascularity of the tip [4]. This design limitation restricts the use of the flap to the ipsilateral nasal ala and immediately adjacent subunits.

As we have illustrated with the above cases, surgical delay is a powerful technique to extend the reach of this workhorse flap. The efficacy of the delay procedure is thought to be mediated by ischaemic pre-conditioning, dilatation of choke vessels, vessel reorientation, and neoangiogenesis [6]. Although surgical delay to improve the survivability of the nasolabial flap is well described, techniques may vary. Unlike other authors who com-

\section{Fig. 4. Versatility of the extended nasolabial flap}

This flap may be used to reconstruct defects as far as the contralateral alar rim (1) and ipsilateral medical canthus (2).

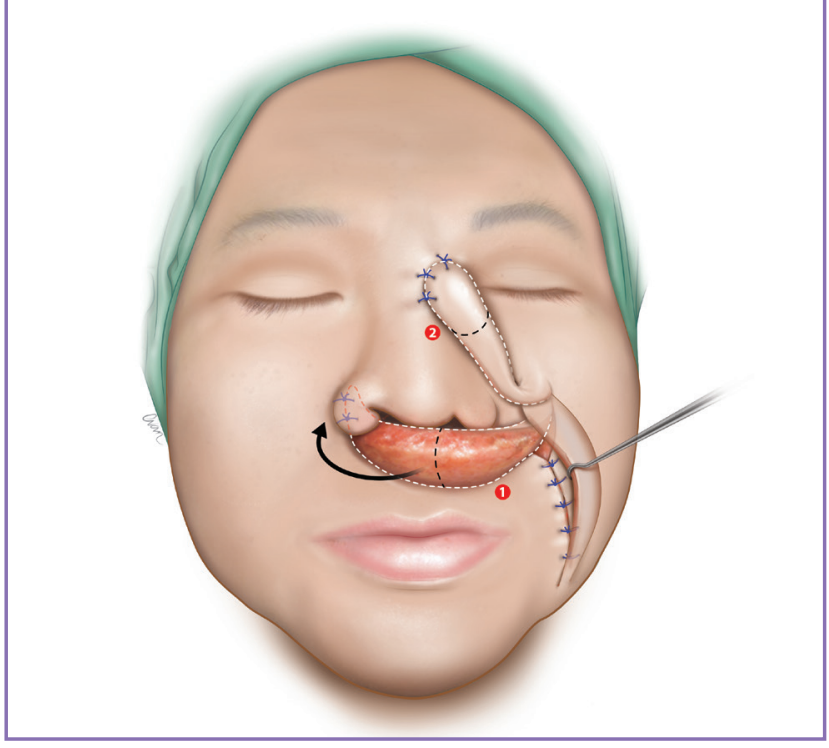

pletely elevate the tip of the flap during the first stage $[3,4,7]$, our technique involved first raising the flap in a bipedicled fashion. Bipedicled flaps offer the advantage of a dual blood supply, which enables a length-to-width ratio exceeding that of a conventional random-pattern flap [2]. Choke vessels can thus open up into the flap from 2 fronts, minimising ischaemia to the distal portion from the outset. This design also prevented retraction of the flap, allowing its length to be maintained as the tip was trained. The bipedicled flap was then converted into an extended unipedicled flap for movement. By dividing the flap progressively, we minimised flap congestion and safeguarded the tip, the crucial portion of the flap. This method allowed us to raise "ultra-long" nasolabial flaps with up to 6:1 length-to-width ratios without any tip necrosis.

Lengthening the reach of the nasolabial flap beyond the ipsilateral ala is advantageous when adjacent skin is unavailable. We successfully reconstructed defects as far as the medial canthal area and contralateral alar base (Fig. 4). Other defects within the radius of this extended flap may also be covered, such as defects of the nasal dorsum or contralateral sidewall, and even intraoral defects. Despite the increase in flap length, the vascularity of the tip was robust enough to withstand aggressive debulking. The flap tip could be safely thinned to the deep dermal layer and folded over on itself for simultaneous resurfacing of both the internal and external linings of full-thickness alar rim defects.

With this technique, flap division was accomplished through several outpatient procedures that were well tolerated by the patients. Preparatory time was increased by a week compared to 
the traditional 2-stage nasolabial flap [8], an acceptable expense in our view to ensure tip survival. Though the resultant scar was longer, extending parallel to the marionette lines, it healed well in our experience and was often imperceptible with minor scar revision.

\section{NOTES}

\section{Conflict of interest}

No potential conflict of interest relevant to this article was reported.

\section{Ethical approval}

The study was approved by the SingHealth Institutional Review Board (IRB No. 2017/2444) and performed in accordance with the principles of the Declaration of Helsinki. Written informed consents were obtained.

\section{Patient consent}

The patients provided written informed consent for the publication and the use of their images.

\section{REFERENCES}

1. Zilinsky I, Alcalay J, Jacobs D, et al. The versatility of the nasolabial flap enhanced by the delay procedure. Dermatol Surg 2001;27:161-4.

2. Darwish AM. Bipedicled flap in reconstruction of exposed tibia. J Plast Reconstr Aesthet Surg 2010;63:160-3.

3. El-Marakby HH. The versatile naso-labial flaps in facial reconstruction. J Egypt Natl Canc Inst 2005; 17:245-50.

4. Weathers WM, Wolfswinkel EM, Nguyen H, et al. Expanded uses for the nasolabial flap. Semin Plast Surg 2013;27: 104-9.

5. Hynes B, Boyd JB. The nasolabial flap: axial or random? Arch Otolaryngol Head Neck Surg 1988;114:1389-91.

6. Ghali S, Butler PE, Tepper OM, et al. Vascular delay revisited. Plast Reconstr Surg 2007;119:1735-44.

7. Durgun M, Ozakpınar HR, Selcuk CT, et al. Repair of fullthickness nasal alar defects using nasolabial perforator flaps. Ann Plast Surg 2015;75:414-7.

8. Menick FJ. The two-stage nasolabial flap for subunit reconstruction of the ala. Oper Tech Plas Reconstr Surg 1998;5: 59-64. 